\title{
The Effect of Organic Stimulant and Inorganic Fertilizer on Two Rice Varieties (Oryza sativa L.)
}

\author{
Erningtyas Widyaswari ${ }^{1 *}$, Mudji Santosa $^{2}$, Moch. Dawam Maghfoer ${ }^{2}$ \\ ${ }^{1}$ Master Program of Agriculture, Facultyof Agriculture, University of Brawijaya, Malang, Indonesia \\ ${ }^{2}$ Department of Plant Science, Faculty of Agriculture, University of Brawijaya, Malang, Indonesia
}

\begin{abstract}
Increased the yield of rice could be done by organic stimulant application and inorganic fertilizer, as well as the used of appropriate variety. The field experiment was aimed to study the interaction of fertilizer application and the using of variety on rice. The research was conducted at April until July 2016 in Sekarputih Hamlet, Pendem Village, Junrejo District, Batu City. This research used the Randomized Complete Block Design (RCBD) Factorial methods with 2 factors are Rice Variety $(\mathrm{V})$ and Fertlizers Doses $(\mathrm{P})$ with 3 replications. Cows Biourine application by dissolved $1 \mathrm{~L}$ biourine with $10 \mathrm{~L}$ water sprayed on soil and plants. EM-4 application was doing by dissolved $100 \mathrm{cc}$ EM-4 with $10 \mathrm{~L}$ water sprayed on soil. The result of research showed that interaction of Mapan-P.05 hybrid variety $\left(V_{2}\right)$ added with fertilizer doses $100 \mathrm{~kg}$ phonska+100 kg urea+cows biourine+EM-4 $\left(P_{6}\right)$ can increase yield on rice in parameter 1000 grain weight to $15.29 \%$ against which added fertilizer doses $200 \mathrm{~kg}$ phonska+200 kg urea $\left(P_{1}\right)$.
\end{abstract}

Keywords: Inorganic Fertilizer, Organic Stimulant, Rice, Variety.

\section{INTRODUCTION}

Rice is the crop plants which produced the rice as the staple food for Indonesian people. Population density increased $1.36 \%$ year by year assumed in 2020, total rice needed are 35.97 million ton with consume assumption is $137 \mathrm{~kg}$ capita $^{-1}[1]$. Two approached for increasing yield of rice are by used hybrid variety and improved cultivation [2]. Hybrid technology reached by heterosis utilization causing a more vigour F1 plant, which can increased to 1 ton ha ${ }^{-1}$; higher than inbreed rice [3].

The balanced fertilization can stimulate theoptimization on the growth of plant [4]. Biourine are plant fertile organic matter made from cow urine and cow faeces with addition of nutrition which used microorganism [5]. Biourine application can enhanced plant height, number of leaves per plant, leaf area and leaf area index as 5.1\%, $6.8 \%, 11.9 \%$ and $10.2 \%$ respectively, higher than without biourine application [6].

To enhance the yield and land productivity, EM-4 (Effective Microorganisme) can be used. It utilize microorganism technology to repair land fertility and soil physics characters [7]. Thus, this study aims to study the interaction between variety of rice and fertilizer to increased the growth and yield of rice.

\footnotetext{
*Correspondence address:

Erningtyas Widyaswari

Email : tyaswidy22@gmail.com

Address : Faculty of Agriculture, University of Brawijaya, Jl. Veteran Malang, 65145
}

\section{MATERIALS AND METHODS}

The research was conducted in April to July 2016, in the Sekarputih Hamlet, Pendem Village, Junrejo District, Batu City. The area elevation is $600 \mathrm{~m}$ above sea level. Soil types is Andisol with $\mathrm{N} 0.13 \%$ (low), C Organic $1.22 \%$ (low), C/N ratio 10 (low), P 6.87 ppm (low), K 0.33 (low) and pH 5.7. Materials used in this study are the seeds of rice variety Ciherang and Mapan-P.05, cow biourine, EM-4, Urea (46\% N), Phonska (15:15:15 NPK) and chemical pesticides. The method used was a Randomized Complete Block Design (RCBD) factorial with 2 factors repeated 3 times.

Table 1. Treatment Methods

\begin{tabular}{ll}
\hline Factor 1 & $\mathrm{~V}_{1}$ : Ciherangrice variety \\
& $\mathrm{V}_{2}$ : Mapan-P.05 rice variety \\
\hline Factor 2 & $\mathrm{P}_{1}:$ : $200 \mathrm{~kg}$ Phonska+200 kg urea ha-1 \\
& $\mathrm{P}_{2}$ : Cow Biourine \\
& $\mathrm{P}_{3}$ : EM-4 \\
& $\mathrm{P}_{4}: 100 \mathrm{~kg}$ Phonska+100 kg urea ha-1 + Cow \\
& Biourine \\
& $\mathrm{P}_{5}: 100 \mathrm{~kg}$ Phonska+100 kg urea ha-1 $+\mathrm{EM}-4$ \\
& $\mathrm{P}_{6}: 100 \mathrm{~kg}$ Phonska+100 kg urea ha-1 + Cow \\
& Biourine + EM-4
\end{tabular}

Land preparation was doing by ploughed with tractor. Land area was $295 \mathrm{~m}^{2}$ made into 36 partition with $3 \times 2 \mathrm{~m}^{2}$ dimension. Manual planting with one seed per hole. Seedlings used rice aged 20 dap (day after planting) or has 3-4 leaves and stem was look bigger.

\section{Inorganic Fertilizer Application}

Fertilizer application was doing at 0 dap with doses of urea are $1 / 4$ doses from total doses. Next 
fertilizing of urea was doing at 20, 40, 60 dap with each doses is $1 / 4$ doses of the total dose. Phonska application was done at 10 dap.

\section{Biourine Application}

Biourine made from fresh urine and feces of cows in the morning. Cows urine were putted on the bottle with dosage $1 \mathrm{~L}$ urine, cows feces were putted on the receptacle with dosage about $5 \mathrm{~kg}$ feces and then added $30 \mathrm{~L}$ water, stired in earlier morning for 7 days. Biourine ready to be used when it scentless and colder than before, and there is a wax layer on the surface of biourine.

Biourine application was doing by dissolved 1 $\mathrm{L}$ biourine with $10 \mathrm{~L}$ water sprayed on soil and plants. Recommendation doses of cow's biourine are $2000 \mathrm{~L} \mathrm{ha}^{-1}$ for three times: at 20 dap as $400 \mathrm{~L}$ ha-1, at 40 dap as $600 \mathrm{~L} \mathrm{ha}^{-1}$, and at 60 dap as 1000 L ha $^{-1}$.

\section{EM-4 Application}

EM-4 application was doing by dissolved 100 cc EM-4 with $10 \mathrm{~L}$ water sprayed on soil. Recommendation doses of EM-4 are $600 \mathrm{~L} \mathrm{ha}^{-1}$ giving by 34 times: at 14 dap as $100 \mathrm{~L} \mathrm{ha}^{-1}$, at 28 dap as 125 $\mathrm{L} \mathrm{ha}{ }^{-1}$, at 42 dap as $175 \mathrm{~L} \mathrm{ha}^{-1}$ and 56 dap as $200 \mathrm{~L}$ $\mathrm{ha}^{-1}$.

Observations made on the growth and the yield of rice plants. For non-destructive observation variables include the high of the plant, number of leaves and number of tillers per plant. Destructive observations include leaf area, leaf area index, dry weight of total plant, crop growth rate and net assimilation rate. The observations were performed at 49, 63, 77 and 91 days after planting. Harvest observations include the number of panicles on the harvested crop, percentage of grain, 1000 grain weight, dry weight of total solar plants, grain weight harvest, and harvest index.

\section{Data Analysis}

Data obtained from observations then analyzed using analysis of variance ( $\mathrm{F}$ test) with level of $5 \%$. If there is a significant difference, then followed by HSD (Honest Significant Difference) test with a level of $5 \%$.

\section{RESULT AND DISCUSSION}

Interaction Influences between Variety Treatment and Adding Fertilizer on Growth and Yield of Rice

There is an interaction on plant height result of Ciherang variety $\left(V_{1}\right)$ respond to a higher plant height with adding fertilizer doses $100 \mathrm{~kg}$ phonska+100 kg urea+cow biourine+EM-4 $\left(\mathrm{P}_{6}\right)$ against cow biourine treatment $\left(P_{2}\right)$. Mapan-P.05 variety $\left(V_{2}\right)$ gave respons to fertilizer doses $100 \mathrm{~kg}$ phon- ska+100 kg urea+cowbiourine $\left(\mathrm{P}_{4}\right)$ and fertilizer doses $100 \mathrm{~kg}$ phonska+100 kg urea+cow biourine+EM-4 $\left(P_{6}\right)$ by produced higher plant height than fertilizer doses $200 \mathrm{~kg}$ phonska+200 kg urea treatment $\left(P_{1}\right)$ (Table 2). Organic matter combination and inorganic fertilizer caused plant growth and yield more higher to $91 \%$ for $\mathrm{Ci}$ herang variety and increased $78 \%$ for hybrid variety than control treatment [8]. Yield of MapanP.05 hybrid variety higher than Ciherang variety are no needed higher measurement and frequency, means higher fertilizer efficiency on hybrid variety [9].

There is an interaction on dry weight total plant result Mapan-P.05 variety $\left(V_{2}\right)$ respons to fertilizer application with doses $100 \mathrm{~kg}$ phonska+100 kg urea+cow biourine+EM-4 $\left(\mathrm{P}_{6}\right)$ produce dry weight total plant higher than EM-4 treatment $\left(P_{3}\right)$ (Table 3). Mapan-P.05 variety if giving organic stimulant and inorganic fertilizer, can produced higher dry weight total plant than just giving EM-4 only. Just Mapan-P.05 hybrid variety from 13 other hybrid variety resulted higher dry weight total plant consistently against which Ciherang variety on some locations at wet or dry season [9].

There is an interaction on 1000 grain weight parameter on Mapan-P.05 variety $\left(\mathrm{V}_{2}\right)$ giving respons to fertilization doses $100 \mathrm{~kg}$ phonska+100 kg urea+cow biourine+EM-4 $\left(\mathrm{P}_{6}\right)$ produced higher 1000 grain weight than fertilizer doses $200 \mathrm{~kg}$ phonska+200 kg urea $\left(P_{1}\right)$ and $100 \mathrm{~kg}$ phonska+100 kg urea+EM-4 treatment $\left(P_{5}\right)$ (Table 4). Total 1000 grain weight influences by environment factors, especially at grain maturity phase. Total 1000 grain weight is the number of biomass consist on grain [10]. Mapan-P.05 variety produce higher 1000 grain weight when added organic stimulant like cow biourine and EM-4, recommend to obtained higher yield of hybrid rice needed to combine inorganic fertilizer (75\%) with organic fertilizer (25\%) on spacing at $20 \mathrm{~cm} \times 20$ $\mathrm{cm}$ [11]. Combination of inorganic fertilizer and organic fertilizer can stablished the sustainability of growth, yield and nutrient uptake hybrid rice [12].

\section{Effect of Variety Treatment to Growth and Yield of Rice}

In the yield of rice, Mapan-P.05 variety produced grain harvest weight and harvest index higher than Ciherang variety $\left(\mathrm{V}_{1}\right)$ (Table 5$)$. Grain harvest weight from this research on Ciherang variety reach 9.54 ton $\mathrm{ha}^{-1}$, whereas on previous study, Ciherang variety resulted higher to 9.90 
ton ha ${ }^{-1}[9]$. For Mapan-P.05 variety in this research, the grain harvest weight reached 13.30 ton $\mathrm{ha}^{-1}$, while previous study for Mapan-P.05 hibryd variety just resulted 10.52 ton ha $^{-1}$ [9]. Thus it can be said that result of this research were higher in hybrid variety Mapan-P.05 than previous study [9].

Effect of Organic Stimulant and Inorganic Fertilizer on Growth and Yield of Rice

Addition of doses fertilizer $100 \mathrm{~kg}$ phonska+100 kg urea+cows biourine $\left(P_{4}\right)$ resulted higher amount of penicles than EM-4 treatment $\left(\mathrm{P}_{3}\right)$ (Table 5). Organic matter such as EM-4 can not produce maximal when apply without adding inorganic fertilizer. The organic matter in the area of study has very low nutrient soil thus less supported to the growth and yield of rice. How- ever, we can repair the physical and biological characteristics of the soil properties [13].

Giving fertilizer with doses $100 \mathrm{~kg}$ phonska+100 kg urea+cows biourine $\left(\mathrm{P}_{4}\right)$ resulted higher grain harvest weight than cows biourine treatment $\left(P_{2}\right)$ (Table 5). Combination of biourine concentration $1 \mathrm{~L}$ urine $+5 \mathrm{~kg}$ feces $+15 \mathrm{~L}$ water $\mathrm{ha}^{-1}$ and inorganic fertilizer with doses $50 \mathrm{~kg} \mathrm{~N}$, $12.5 \mathrm{~kg} \mathrm{P}_{2} \mathrm{O}_{5}, 17.5 \mathrm{~kg} \mathrm{~K} \mathrm{~K}_{2} \mathrm{O}$ resulting amount of tuber on shallot increased to $27.33 \%$ than without biourine concentration and inorganic fertilizer doses [4]. Giving organic fertilizer 50\% and inorganic fertilizer $50 \%$ produced optimal growth and yield on rice [14]. Organic fertilizer can reduce the inorganic fertilizer uses as $25 \%$ on rice field, by not decreasing the growth and yield on rice. It also can repairs the physical, biological and chemical soil properties [15].

Table 2. Average Plant Height per plant on rice result due to interaction Variety Different and Adding Fertilizer at 35 DAP

\begin{tabular}{lcccccc}
\hline \multirow{2}{*}{ Rice Variety } & \multicolumn{5}{c}{ Plant Height (cm) } \\
\cline { 2 - 6 } & $\mathbf{P}_{\mathbf{1}}$ & $\mathbf{P}_{\mathbf{2}}$ & $\mathbf{P}_{\mathbf{3}}$ & $\mathbf{P}_{\mathbf{4}}$ & $\mathbf{P}_{\mathbf{5}}$ & $\mathbf{P}_{\mathbf{6}}$ \\
\hline Ciherang $\left(\mathrm{V}_{1}\right)$ & $40.83 \mathrm{abcd}$ & $37.33 \mathrm{a}$ & $38.91 \mathrm{ab}$ & $38.91 \mathrm{ab}$ & $39.58 \mathrm{abc}$ & $44.92 \mathrm{bcde}$ \\
Mapan-P.05 $\left(\mathrm{V}_{2}\right)$ & $43.25 \mathrm{abcd}$ & $48 \mathrm{de}$ & $46.75 \mathrm{cde}$ & $51.25 \mathrm{e}$ & $47.67 \mathrm{de}$ & $50.83 \mathrm{e}$ \\
\hline HSD 5\% & & & 7.27 & \\
\hline CV (\%) & & & 5.56 &
\end{tabular}

Notes: Numbers with same letters are not significantly different at $5 \%$ level using HSD test, CV= Coefficient of Variation

$\mathrm{P}_{1}=200 \mathrm{~kg}$ Phonska+200 kg urea $\quad \mathrm{P}_{4}=100 \mathrm{~kg}$ Phonska+100 kg urea + Cow Biourine

$P_{2}=$ Cow Biourine $\quad P_{5}=100 \mathrm{~kg}$ Phonska $+100 \mathrm{~kg}$ urea + EM -4

$P_{3}=E M-4 \quad P_{6}=100 \mathrm{~kg}$ Phonska+100 kg urea + Cow Biourine + EM-4

Table 3. Average Dry Weight Total Plant per plant on rice result due to Variety Different and Adding Fertilizer at 91 DAP

\begin{tabular}{|c|c|c|c|c|c|c|}
\hline \multirow{2}{*}{ Rice Variety } & \multicolumn{6}{|c|}{ Dry Weight Total Plant (g) } \\
\hline & $\mathbf{P}_{1}$ & $\mathbf{P}_{2}$ & $\mathbf{P}_{3}$ & $\mathbf{P}_{4}$ & $P_{5}$ & $\mathbf{P}_{6}$ \\
\hline Ciherang $\left(\mathrm{V}_{1}\right)$ & $121.23 \mathrm{abc}$ & $102.78 \mathrm{a}$ & $108.51 \mathrm{ab}$ & 126.08 abcd & 137.23 abcde & 152.29 abcde \\
\hline HSD 5\% & \multicolumn{6}{|c|}{51.37} \\
\hline CV (\%) & \multicolumn{6}{|c|}{12.38} \\
\hline
\end{tabular}

Notes: Numbers with same letters are not significantly different at 5\% level using HSD test, CV= Coefficient of Variation

$\mathrm{P}_{1}=200 \mathrm{~kg}$ Phonska+200 kg urea $\quad \mathrm{P}_{4}=100 \mathrm{~kg}$ Phonska+100 kg urea + Cow Biourine

$P_{2}=$ Cow Biourine $\quad P_{5}=100 \mathrm{~kg}$ Phonska $+100 \mathrm{~kg}$ urea + EM-4

$P_{3}=$ EM-4 $\quad P_{6}=100 \mathrm{~kg}$ Phonska $+100 \mathrm{~kg}$ urea + Cow Biourine + EM -4

Table 4. Average 1000 grain Weight per plant on rice result due to Variety Different and Adding Fertilizer at 115 DAP

\begin{tabular}{|c|c|c|c|c|c|c|}
\hline \multirow{2}{*}{ Rice Variety } & \multicolumn{6}{|c|}{1000 Grain Weight (g) } \\
\hline & $\mathbf{P}_{1}$ & $\mathbf{P}_{\mathbf{2}}$ & $\mathbf{P}_{3}$ & $\mathbf{P}_{4}$ & $\mathbf{P}_{5}$ & $\mathbf{P}_{6}$ \\
\hline Ciherang $\left(\mathrm{V}_{1}\right)$ & $27.52 \mathrm{ab}$ & $27.14 \mathrm{a}$ & $26.68 \mathrm{abc}$ & $27.67 \mathrm{abc}$ & $29.12 \mathrm{abc}$ & $28.42 \mathrm{abc}$ \\
\hline HSD 5\% & & & & 3.87 & & \\
\hline CV (\%) & & & & 4.39 & & \\
\hline
\end{tabular}

Notes: Numbers with same letters are not significantly different at 5\% level using HSD test, CV= Coefficient of Variation

$P_{1}=200 \mathrm{~kg}$ Phonska+200 kg urea $\quad P_{4}=100 \mathrm{~kg}$ Phonska+100 kg urea + Cow Biourine

$\mathrm{P}_{2}=$ Cow Biourine $\quad \mathrm{P}_{5}=100 \mathrm{~kg}$ Phonska+100 kg urea $+\mathrm{EM}-4$

$P_{3}=$ EM-4 $\quad P_{6}=100 \mathrm{~kg}$ Phonska $+100 \mathrm{~kg}$ urea + Cow Biourine + EM -4

\section{Soil conditions}

C-Organic and $\mathrm{N}$-total increased at all fertilizer treatment (Table 6). Higher C-Organic available on treatment fertilizer doses 100 kg phon- ska+100 kg urea+cows biourine $\left(\mathrm{P}_{4}\right)$ increased to $37.70 \%$ compared with before treatment. Higher $\mathrm{N}$-total available on treatment fertilizer doses $100 \mathrm{~kg}$ phonska+100 kg urea+cows biourine+EM- 
$4\left(P_{6}\right)$ increased $61.54 \%$ compared with before treatment. It is caused by the addition of $\mathrm{N}$ ferti- lizer with high dosages which provide higher $\mathrm{N}$ total on soil [16].

Table 5. Average Yield of Rice for each treatment Variety Different and Adding Fertilizer

\begin{tabular}{|c|c|c|c|c|}
\hline \multirow[b]{2}{*}{ Treatments } & \multicolumn{4}{|c|}{ Average Yield of Rice } \\
\hline & $\begin{array}{c}\text { Amount } \\
\text { of } \\
\text { Panicles }\end{array}$ & $\begin{array}{c}\text { Grain } \\
\text { Percentage } \\
\text { (\%) }\end{array}$ & $\begin{array}{c}\text { Grain } \\
\text { Harvest } \\
\text { Weight } \\
\text { (t ha-1) }\end{array}$ & $\begin{array}{c}\text { Harvest } \\
\text { Index } \\
(\%)\end{array}$ \\
\hline \multicolumn{5}{|l|}{ Rice Variety } \\
\hline Ciherang $\left(\mathrm{V}_{1}\right)$ & 17.1 & 83.5 & $9.54 \mathrm{a}$ & $27.83 \mathrm{a}$ \\
\hline Mapan-P.05 $\left(V_{2}\right)$ & 17.08 & 79.8 & $13.30 \mathrm{~b}$ & $33.17 \mathrm{~b}$ \\
\hline HSD 5\% & ns & ns & 2.38 & 4.74 \\
\hline \multicolumn{5}{|l|}{ Adding Fertilizer $\left(\mathrm{ha}^{-1}\right)$} \\
\hline 200 kg Phonska+200 kg urea $\left(P_{1}\right)$ & $17.94 \mathrm{ab}$ & 85.31 & $12.51 \mathrm{ab}$ & 29.5 \\
\hline Cows Biourine $\left(P_{2}\right)$ & $15.44 \mathrm{ab}$ & 78.98 & $8.92 \mathrm{a}$ & 27.45 \\
\hline $\mathrm{EM}-4\left(\mathrm{P}_{3}\right)$ & $14.36 \mathrm{a}$ & 83.13 & $10.01 \mathrm{ab}$ & 29.29 \\
\hline 100 kg Phonska+100 kg urea + Cows Biourine $\left(P_{4}\right)$ & $19.81 \mathrm{~b}$ & 83.76 & $13.12 \mathrm{~b}$ & 32.84 \\
\hline 100 kg Phonska+100 kg urea + EM-4 $\left(P_{5}\right)$ & $17.47 \mathrm{ab}$ & 75.11 & $12.46 \mathrm{ab}$ & 29.6 \\
\hline 100 kg Phonska+100 kg urea + Cows Biourine $+E M-4\left(P_{6}\right)$ & $17.53 \mathrm{ab}$ & 83.6 & $11.51 \mathrm{ab}$ & 34.33 \\
\hline HSD 5\% & 4.52 & ns & 4.12 & tn \\
\hline CV (\%) & 12.58 & 7.26 & 17.21 & 12.83 \\
\hline
\end{tabular}

Note : Numeral within columns followed by same letters are not significantly different at 5\% level using HSD test; ns:non significant; $\mathrm{CV}=$ Coeffiecient of Variation

Table 6. Soil Analysis Before and After Treatments

\begin{tabular}{lcccccc}
\hline \multicolumn{1}{c}{ Parameters } & $\mathbf{p H}$ & $\mathbf{C}$-Organic (\%) & $\mathbf{N}$-Total $\mathbf{( \% )}$ & $\mathbf{C} / \mathbf{N}$ & $\mathbf{P}\left(\mathbf{m g ~ k g}^{-1}\right)$ & $\mathbf{K}\left(\mathbf{m e ~} \mathbf{~ 1 0 0 ~}^{-\mathbf{1}}\right)$ \\
\hline Before treatment & 5.7 & 1.22 & 0.13 & 10 & 6.87 & 0.33 \\
\hline After treatment & & & & & & \\
\hline $\mathrm{V}_{1} \mathrm{P}_{1}$ & 5.3 & 1.35 & 0.17 & 8 & 3.03 & 0.23 \\
$\mathrm{~V}_{1} \mathrm{P}_{2}$ & 5.3 & 1.36 & 0.14 & 9 & 7.63 & 0.35 \\
$\mathrm{~V}_{1} \mathrm{P}_{3}$ & 5.4 & 1.36 & 0.15 & 9 & 1.53 & 0.42 \\
$\mathrm{~V}_{1} \mathrm{P}_{4}$ & 5.9 & 1.68 & 0.17 & 10 & 11.33 & 0.41 \\
$\mathrm{~V}_{1} \mathrm{P}_{5}$ & 5.5 & 1.27 & 0.15 & 8 & 3.81 & 0.29 \\
$\mathrm{~V}_{1} \mathrm{P}_{6}$ & 5.4 & 1.53 & 0.16 & 10 & 2.29 & 0.44 \\
\hline $\mathrm{V}_{2} \mathrm{P}_{1}$ & 5.6 & 1.28 & 0.15 & 8 & 1.53 & 0.22 \\
$\mathrm{~V}_{2} \mathrm{P}_{2}$ & 5.6 & 1.44 & 0.16 & 9 & 2.28 & 0.41 \\
$\mathrm{~V}_{2} \mathrm{P}_{3}$ & 5.9 & 1.34 & 0.17 & 8 & 1.51 & 0.43 \\
$\mathrm{~V}_{2} \mathrm{P}_{4}$ & 5.6 & 1.27 & 0.15 & 8 & 1.52 & 0.32 \\
$\mathrm{~V}_{2} \mathrm{P}_{5}$ & 6.0 & 1.51 & 0.17 & 9 & 2.27 & 0.51 \\
$\mathrm{~V}_{2} \mathrm{P}_{6}$ & 5.6 & 1.66 & 0.21 & 8 & 3.14 & 0.36 \\
\hline
\end{tabular}

\section{CONCLUSION}

There is an interaction on Mapan-P.05 hybrid variety with fertilizer doses $100 \mathrm{~kg}$ phonska+100 $\mathrm{kg}$ urea+cows biourine+EM-4 on yield parameters are 1000 grain weight with percentage of increase as $15.29 \%$ compare with fertilizer doses 200 kg phonska+200 kg urea. Mapan-P.05 hybrid variety gives the significant influences on grain harvest weight and harvest index; each increase percentage as $39.41 \%$ and $27.83 \%$ compared with Ciherang variety. Fertilizer doses $100 \mathrm{~kg}$ phonska+100 kg urea+cows biourine increasing amount of panicles and grain harvest weight with increase percentage as $37.95 \%$ and $47.08 \%$ compare with EM- 4 and cows biourine treatment.

\section{REFERENCES}

[1] Irianto, G.S. 2009. Peningkatan produksi padi melalui IP Padi 400. Indonesian Center for Rice Research. Indonesian Agency for Agricultural Research and Development. Jakarta.

[2] Satoto dan Rumanti. 2011. Galur mandul Jantan untuk Perakitan padi hibrida. Jurnal Iptek Tanaman Pangan. 6(1). 14-29.

[3] Virmani, S.S., I. Kumar. 2004. Development and use of hybrid rice technology to increase rice productivity in the tropic. Int. Rice. Res. Note. 19(1). 10-19.

[4] Widyaswari, E. 2016. Pengaruh biourin sapi dan pupuk anorganik pada tanaman bawang merah (Allium ascalonicum L.). 
Bachelor Thesis. Faculty of Agriculture. University of Brawijaya. Malang.

[5] Wati, Y.T., E.E. Nurlaelih, M. Santosa. 2014. Pengaruh aplikasi biourin pada pertumbuhan dan hasil tanaman bawang merah (Allium ascalonicum L.). Jurnal Produksi Tanaman. 2(8). 613 - 619.

[6] Santosa, M., M.D. Maghfoer, S. Fajriani. 2014. The effect of solid fertilizers and biourine application on plants rice $\mathrm{Cv}$ Ciherang at Ngujung, Batu, East Java. Res. J. Life Sci. 1(2). 146-153.

[7] Yulhasmir. 2009. Konsentrasi EM4 (Effective Microorganisme) dan jarak tanam terhadap pertumbuhan dan produksi tanaman jagung (Zea mays L.) dengan sistem tanpa olah tanah. Jurnal Agronobis. 1(1). 1-11.

[8] Abdulrrachman, S. 2007. Komparatif berbagai metode penetapan kebutuhan pupuk pada tanaman padi. Seminar on Appreciation towards Rice Research. Jakarta. 115-125.

[9] Suyamto, M. Saeri, D.P. Saraswati, Robi'in. 2015. Verifikasi dosis rekomendasi pemupukan hara spesifik lokasi untuk padi varietas hibrida. Jurnal Penelitian Pertanian Tanaman Pangan. 34(3). 165-174.

[10] Jannah, A., Y.S. Rahayu, K. Sulanjari. 2012. Respon pertumbuhan dan produksi padi varietas Ciherang pada pemberian kombinasi dosis pupuk anorganik dan pupuk kandang ayam. Institute of Research and Community Service, Singaperbangsa University. Karawang.

[11] Bezbaruha, R., R.C. Sharma, P. Banik. 2011. Effect of nutrient management and planting geometry on productivity of hybrid rice cultivars. Am. J. Plant Sci. 2. 297-302.

[12] Pandey, D., D.K. Payasi, N. Pandey. 2014. Effect of organic and inorganic fertilizers on hybrid rice. Int. J. Current Res. 6(5). 65496551.

[13] Kariada, I.K., I.B. Aribawa. 2006. Pengaruh residu jenis dan dosis pupuk organik terhadap pertumbuhan dan hasil padi di Bali. Research Report. Research and Development, Department of Agriculture, Bali.

[14] Saidah, D. Bulo, Syafruddin. 2006. Pemanfaatan pupuk kandang dan anorganik pada padi sawah dalam system integrasi Padi-Ternak di Sulawesi Tengah. Jurnal Agribisnis. 7(2). 95-100.

[15] Amilia, Y. 2011. Penggunaan pupuk organik cair untuk mengurangi dosis penggunaan pupuk anorganik pada padi sawah (Oryza sativa L.). Bachelor Thesis. Faculty of Agriculture. Bogor Agricultural University. Bogor.

[16] Firmansyah, I., N. Sumarni. 2013. Pengaruh dosis pupuk $\mathrm{N}$ dan varietas terhadap $\mathrm{pH}$ tanah, $\mathrm{N}$-total tanah, serapan $\mathrm{N}$, dan hasil umbi bawang merah pada tanah entisols Brebes Jawa Tengah. Jurnal Hortikultura. 23(4). 358-364. 